\title{
Knowledge, Behavioural Response and Anxiety in Relation to COVID-19 Outbreak Among Nepalese Adult: A Cross Sectional Electronic Survey
}

\author{
Biswash Sapkota $^{1}$, Bibechana Sapkota $^{2,}$, , Bishal Sapkota ${ }^{3}$, Nirmal Lamichhane ${ }^{4}$ \\ ${ }^{1}$ Department of Pharmacy, Novel Academy, Pokhara, Nepal \\ ${ }^{2}$ School of Health and Allied Sciences, Pokhara University, Pokhara, Nepal \\ ${ }^{3}$ Department of Emergency Medicine, Lekhnath City Hospital Pvt LTD, Pokhara, Nepal \\ ${ }^{4}$ Department of Neuropsychiatry, Gandaki Medical College Teaching Hospital and Research Center (P) Ltd, Pokhara, Nepal
}

Email address:

merobiswash8@gmail.com (Biswash S.), bibechanasapkota@gmail.com (Bibechana S.), dr.bishalsapkota@gmail.com (Bishal S.), drnrmlam@hotmail.com (Nirmal L.)

*Corresponding author

\section{To cite this article:}

Biswash Sapkota, Bibechana Sapkota, Bishal Sapkota, Nirmal Lamichhane. Knowledge, Behavioural Response and Anxiety in Relation to COVID-19 Outbreak Among Nepalese Adult: A Cross Sectional Electronic Survey. American Journal of Psychiatry and Neuroscience.

Vol. 8, No. 4, 2020,pp. 70-77. doi: 10.11648/j.ajpn.20200804.12

Received: October 15, 2020; Accepted: November 2, 2020; Published: November 19, 2020

\begin{abstract}
Coronavirus disease (COVID-19) is an infectious disease caused by a newly discovered coronavirus. It has its severe effects on those effected but those people who are not infected might also suffer from various disorders that includes mental disorders also. A cross-sectional study was carried out from April 20, 2020, among the adult people during the strict lockdown period in Nepal. The survey was online using google forms. The form was distributed through Facebook groups and Messenger. Nepali nationals, over 18 years of age, who agreed to participate in the study, were asked to complete the questionnaire by clicking on the provided link. A total of 383 participants completed the questionnaire. The mean age group was 25.45 years (SD: 7.49$), 195(50.9 \%)$ were male, and $188(49.1 \%)$ were female. The majority of the respondent $(80.7 \%)$ had better knowledge on COVID-19, 70.2\% reported taking the avoidance behaviour and almost all (99\%) were reported taking anyone and more than one of the recommended behaviour. Females were more likely to take avoidance behaviour than men ( $\mathrm{AOR}=2.549,95 \%$ C.I: $1.608-4.043$ ). General anxiety disorder was reported on $12.5 \%$ of the total respondents. Respondents showing avoidance behaviour, having children and having serious illness had a significantly higher level of anxiety. The study shows that people were more conscious regarding the impact of the pandemic but few people developed anxiety. The preventive measures should be taken to prevent the people from further post-traumatic stress disorder and other mental issues.
\end{abstract}

Keywords: Anxiety, Behavioural Response, Online Survey, Knowledge

\section{Introduction}

Pandemic situation like COVID-19 outbreaks arise the fear related behaviour among people and there is always an increased risk of mental health disorders. In the absence of appropriate medical interventions, the main method of controlling outbreaks is to change public behaviour [1]. An individual's behaviour can affect their family, social networks, organizations, communities and information they obtain and the impact on their society. The fear and anxiety related to epidemics and pandemics also influence the behaviour of people in the community [2].

A research in India shows that participant have moderate level of knowledge about COVID 19 while they have adequate knowledge regarding the preventive aspects. The anxiety level of the participants was high and most of the participants perceived mental health care need. The awareness to the people was necessary to address the mental health issues [1]. A study shows that nearly $50 \%$ of the 
participants have high level of anxiety and $48 \%$ feels lonely during social distancing. In case, of married man they have high level of anxiety than the unmarried man [3].

In this context, understanding the general publics' knowledges, behavioural and anxious level could provide useful information to policy makers, enabling the planning of educational interventions on the population to effectively curb the outbreak. Therefore, to address the gap in the literature, the primary objective of the present investigation was to examine the level of knowledge, behavioural responses, and anxiety levels of people in mainland Nepal during the ongoing COVID-19 outbreak.

\section{Materials and Method}

\subsection{Study Design and Participants}

We commenced 15 days (April 20 to May 5, 2020) crosssectional, web-based anonymous survey using an online questionnaire. The survey link was advertised in Facebook groups and Messenger, the most popular and widely used social media platform in Nepal. The inclusion criteria were that the respondents were mainland Nepal residents who were between 18 and 70 years of age. Prior to accessing the survey, participants read an informed consent statement that described that participation was voluntary and that they could stop at any time. By clicking on a "next" button, participants were informed that they were providing consent to complete the survey. The questionnaire was developed in English and was then translated into Nepalese. Local experts validated the content of the questionnaire, after which it was pilot tested. The survey consisted of four sections, which assessed (1) Sociodemographic section, (2) knowledge about COVID-19, (3) behavioural responses towards COVID-19 outbreak (4) General anxiety disorder due to COVID-19 outbreak.

\subsection{Knowledge}

The participants' knowledge was assessed using a series of questions regarding signs and symptoms of COVID-19 infection, transmission, and protection from infection. The questionnaire contains 7 questions, including clinical presentations, transmission routes and prevention and control methods of COVID-19. A correct response was given a score of one and an incorrect or "don't know" response was scored zero. The possible total knowledge score ranged from 0 to 7 , with higher scores representing higher levels of knowledge. Mean knowledge score was calculated, based on the mean knowledge score participants with scores $\geq$ mean points denote better knowledge of COVID-19.

\subsection{Behavioural Response}

Participants were asked eight questions about recent behaviour. Six related to avoidance of places or activities, behaviour that had not been forcibly recommended by the government. Two related to activities that had been recommended - namely, increased cleaning or disinfecting of surfaces, washing hands with soap and water more often than usual. Permitted responses for each question were yes, no, or does not apply. In case of avoidance behaviour "yes" response was given score of one and a no or does not apply response was scored zero. The possible total avoidance behaviour score ranged from 0-6. Mean avoidance behaviour score was than calculated, based on the mean avoidance behaviour score participants with scores $\geq$ mean points were assumed taking avoidance behaviour of COVID-19. Regarding, recommended behaviour "yes" response was given score of one and no or does not apply response was scored zero. The possible total recommended behaviour score ranged from 0-2. Respondent with recommended behaviour score $\geq 1$ assumed taking recommended behaviour. As a supplementary question we also asked "In the past 24 hours, how many times have you washed your hands with soap and water? That does not include having a shower, a bath, or doing the washing up [4].

\subsection{Anxiety Symptoms}

We used Chinese version of GAD-7 (Generalized Anxiety Disorder-7) scale to assess subject's anxiety symptoms. The GAD-7 has been previously used in Chinese populations, and found to have good reliability (Cronbach's alpha=0.90) [5]. Seven items assessed the frequency of anxiety symptoms over the past two weeks on a 4-point Likert-scale ranging from 0 (never) to 3 (nearly every day). The total score of GAD-7 ranged from 0 to 21 , with increasing scores indicating more severe functional impairments as a result of anxiety [6]. For the purpose of this study, we defined a GADtotal score of 9 points or greater as the presence of anxiety symptoms [7].

\subsection{Ethical Consideration}

This study protocol was approved by ethical committee of Nepal Health Research Council (NHRC), Nepal before the commencement of study.

\subsection{Statistical Analysis}

The collected data were analyzed using SPSS version 16.0. The data analysis was done by frequency run and cross tab. In bivariate analysis chi-square test was done and in multivariate analysis binary logistic regression analysis was done to find out the association between outcome and explorative variables. Odd ratios and corresponding 95\% CI were used to find out the significant of associations. $\mathrm{P}<0.05$ was considered statistically significant.

\section{Results}

\subsection{Respondents Demographic Characteristics}

As shown in Table 1, total of 383 sample were analyzed and above half $(50.9 \%)$ of the respondents were male and more than $(52.5 \%)$ were in the age group of 18 to 24 years old and a mean age of $25.42 \pm 7.27$ years. Concerning the ethnicity, $80.4 \%$ of the respondents were Brahmin and 
Chhetri followed by other ethnic's groups. Regarding the education, $61.6 \%$ of the respondents were graduate students followed by $18.3 \%$ respondents completed secondary level. Most of the respondents were unmarried $(77.3 \%)$, regarding chronic illness few respondents $(6.3 \%)$ have chronic illness like diabetes, hypertension, gastritis. In case of occupation most of the respondent were students (60.6), followed by most of the respondent doing private job (20.9), few running their own business and government job.

Table 1. Demographic characteristics of respondents $(N=383)$.

\begin{tabular}{ll}
\hline Demographic characteristics & $\mathbf{N}(\%)$ \\
\hline Gender & $195(50.9)$ \\
Male & $188(49.1)$ \\
Female & \\
Age & $201(52.5)$ \\
$18-24$ & $158(41.3)$ \\
$25-34$ & $11(2.9)$ \\
$35-44$ & $5(1.3)$ \\
$45-54$ & $8(2.1)$ \\
$55-64$ & \\
Ethnicity & $308(80.4)$ \\
Brahmin/Chhetri & $75(19.6)$ \\
Other than Brahmin/Chhetri & \\
Education & $8(2.1)$ \\
Primary & $70(18.3)$ \\
Secondary & $236(61.6)$ \\
Graduate & $64(16.7)$ \\
Post-graduate & $5(1.3)$ \\
Doctorate & \\
Marital status & $87(22.7)$ \\
Married & $296(77.3)$ \\
Unmarried & \\
Chronic illness & $24(6.3)$ \\
Yes & $359(93.7)$ \\
No & \\
Occupation & $232(60.6)$ \\
Student & $80(20.9)$ \\
Private job & $18(4.7)$ \\
Government job & $16(4.2)$ \\
Business & $37(9.7)$ \\
Others &
\end{tabular}

\subsection{Respondents Level of Knowledge Regarding COVID-19} Pandemic

Figure 1 describes the level of knowledge among the respondent regarding COVID-19 pandemic and Table 2 shows the proportion of correct responses to knowledge items. The participants' knowledge level was assessed using seven questions regarding signs and symptoms of COVID-19 infection, transmission, and protection from infection. As shown in Figure 1, the most $(80.7 \%)$ of respondents had an adequate knowledge level regarding COVID-19 pandemic. The mean COVID-19 knowledge score was 6.1 (SD: 1.6, range: $0-7)$, suggesting an overall $87 \%(6.1 / 7 * 100)$ correct rate on this knowledge level test. The lowest percentages (77.8\%) know about N-95 mask which is suitable for COVID-19 pandemic.
Table 2. Responses to the Knowledge items.

\begin{tabular}{|c|c|}
\hline Symptoms & $\mathbf{N}(\%)$ \\
\hline Fever & $43(11.2)$ \\
\hline Dry cough & $8(2.1)$ \\
\hline Shortness of breath & $2(0.5)$ \\
\hline All of above & $330(86.2)$ \\
\hline \multicolumn{2}{|l|}{ Mode of Transmission } \\
\hline Person to person through small droplets & $16(4.2)$ \\
\hline Touching contaminated surface & $8(2.1)$ \\
\hline $\begin{array}{l}\text { Breathe in droplets from a person with } \\
\text { COVID- } 19 \text { who coughs out or exhales }\end{array}$ & $4(1.0)$ \\
\hline \multicolumn{2}{|l|}{ Incubation Period of COVID-19 } \\
\hline 2-14 days & $365(95.3)$ \\
\hline 24 days & $13(3.4)$ \\
\hline 30 days & $1(0.3)$ \\
\hline 35 days & $4(1.0)$ \\
\hline \multicolumn{2}{|l|}{ Availability of Medicine } \\
\hline Yes & $14(3.7)$ \\
\hline No & $340(88.8)$ \\
\hline Don’t Know & $29(7.6)$ \\
\hline \multicolumn{2}{|l|}{ Quarantine Means } \\
\hline Infected people & $26(6.8)$ \\
\hline Healthy people & $6(1.6)$ \\
\hline People who are at risk & $327(85.4)$ \\
\hline Everyone & $24(6.3)$ \\
\hline \multicolumn{2}{|l|}{ Isolation Means } \\
\hline Infected people & $342(89.3)$ \\
\hline Healthy people & $9(2.3)$ \\
\hline People who are at risk & $17(4.4)$ \\
\hline Everyone & $15(3.9)$ \\
\hline \multicolumn{2}{|l|}{ Types of Face Mask Suitable } \\
\hline Surgical mask & $39(10.2)$ \\
\hline N-95 face mask & $2989(77.8)$ \\
\hline Flu face mask & $2(0.5)$ \\
\hline Cotton face mask & $44(11.5)$ \\
\hline
\end{tabular}

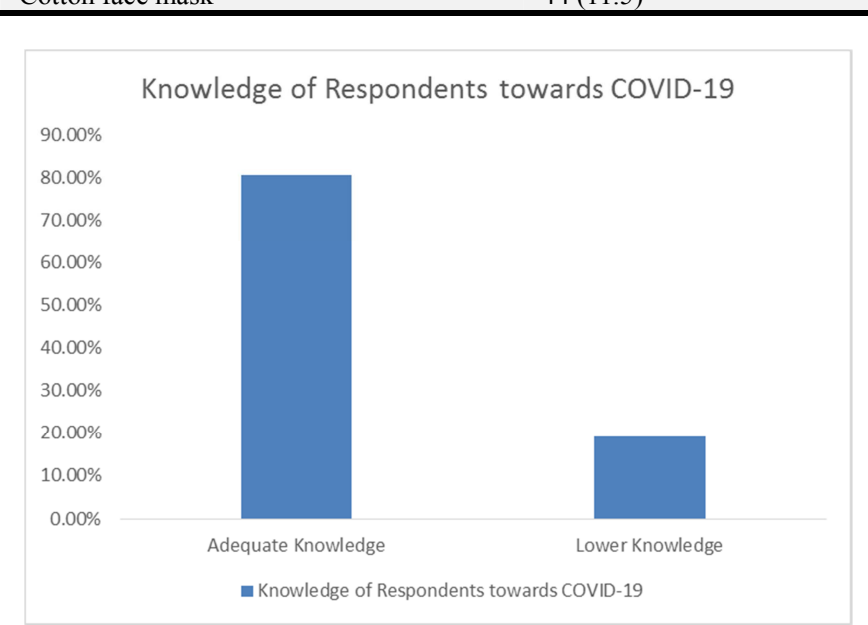

Figure 1. Respondents Level of Knowledge regarding COVID-19 Pandemic.

\subsection{Respondents Behavioural Response Towards COVID-19 Pandemic}

Figure 2 demonstrates the level of behaviour towards COVID-19 pandemic among the respondents and Table 3 shows the proportion towards behavioural responses. The level of respondents' behaviour toward COVID-19 pandemic was assessed via eight questions out of which six related to avoidance of places or activities, behaviours that had not been recommended by the government, while two related to 
activities that had been recommended by government followed with "Yes", "No" or Not "Applicable" choices. As shown in Figure 2, the findings indicating that the majority of the total respondents $70.2 \%$ reported taking the avoidance behaviour and almost all i.e. $99 \%$ of them reported that they were taking any one and more than one of the recommended behaviour. It shows that due to the pandemic situation $70.2 \%$ of the people have taken time off from work, $49.3 \%$ people haven't send their children to the school, $86.2 \%$ people have cleaned there houses more often than previous and $98.2 \%$ people are using soap and water to wash hand regularly, $42.3 \%$ of the people wash their hands with soap and water for 4-9 times a days during this pandemic situation.

Table 3. Behavioural Responses to Corona Outbreak.

\begin{tabular}{|c|c|c|c|}
\hline Activities & Yes $(\%)$ & No $(\%)$ & Not Applicable \\
\hline $\begin{array}{l}\text { Deliberately cancelled or postponed a social event, such as meeting friends, eating out, or going to a } \\
\text { sports event* }\end{array}$ & $348(90.9)$ & $21(5.5)$ & $14(3.7)$ \\
\hline Reduced the amount I use public transport* & $341(89)$ & $17(4.4)$ & $25(65)$ \\
\hline Taken time off work* & $269(70.2)$ & $57(14.9)$ & $57(14.9)$ \\
\hline Reduced the amount I go into shops* & $362(94.5)$ & $11(2.9)$ & $10(2.6)$ \\
\hline Kept one or more of my children out of school or nursery* & $189(49.3)$ & $10(2.6)$ & $184(48)$ \\
\hline Kept away from crowded places generally* & $378(98.7)$ & $2(0.5)$ & $3(0.8)$ \\
\hline Washed my hands with soap and water more often than usual ${ }^{+}$ & $376(98.2)$ & $6(1.6)$ & $1(0.3)$ \\
\hline
\end{tabular}

(* indicates the avoidance behaviour, + indicates the recommended behaviour).

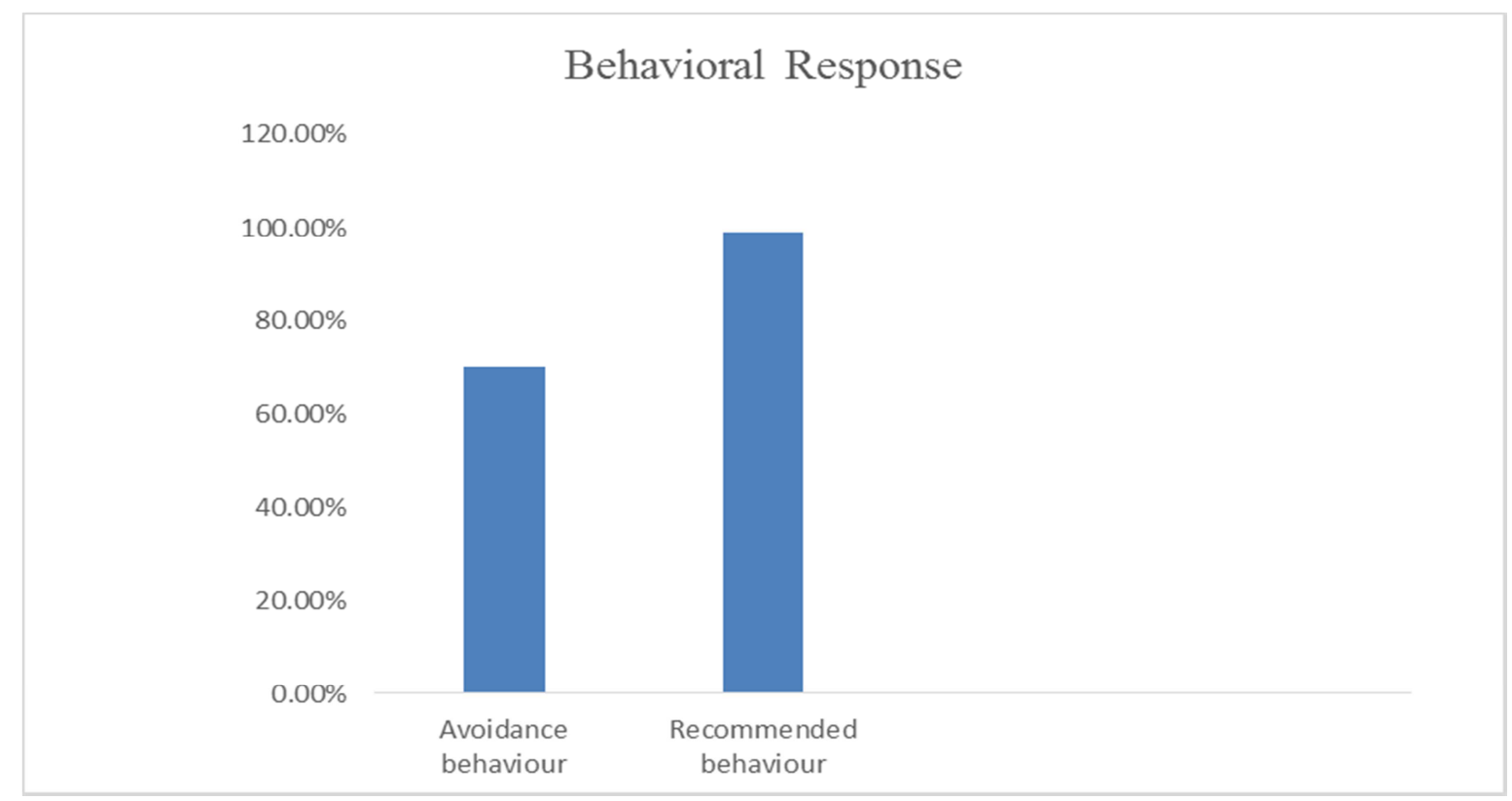

Figure 2. Respondents Behavioural Response towards COVID-19 Pandemic.

\subsection{Respondents Anxiety Towards COVID-19 Pandemic}

Figure 3 presents the level of anxiety among respondents toward COVID-19 pandemic and Table 4 shows the proportion towards general anxiety disorder. Level of the respondents' anxiety was assessed via Chinese version of GAD-7 scale. As shown in Figure 3, the findings indicate that $12.5 \%$ of the respondents had anxiety and other respondents don't have anxiety.

Table 4. Responses towards GAD due to COVID-19.

\begin{tabular}{llll}
\hline $\begin{array}{l}\text { Over the last } \mathbf{2} \text { weeks, how often have you been bothered } \\
\text { by the following problems }\end{array}$ & $\begin{array}{l}\text { Not at all sure } \\
\mathbf{( \% )}\end{array}$ & Several days $(\%)$ & $\begin{array}{l}\text { Over half the } \\
\text { days }(\%)\end{array}$ \\
\hline Feeling nervous, anxious, or on edge & $261(68.1)$ & $75(19.6)$ & $22(5.7)$ \\
Not being able to stop or control worrying & $259(67.6)$ & $68(17.8)$ & $25(6.3)$ \\
Worrying too much about different things & $246(64.2)$ & $75(19.6)$ & $26(6.8)$ \\
Trouble relaxing & $252(65.8)$ & $75(19.6)$ & $34(8.9)$ \\
Being so restless that it's hard to sit still & $268(70.0)$ & $72(18.8)$ & $28(7.3)$ \\
Becoming easily annoyed or irritable & $249(65.0)$ & $94(24.5)$ & $22(57.0)$ \\
Feeling afraid as if something awful might happen & $224(58.5)$ & $128(33.4)$ & $18(9.4)$ \\
\hline
\end{tabular}




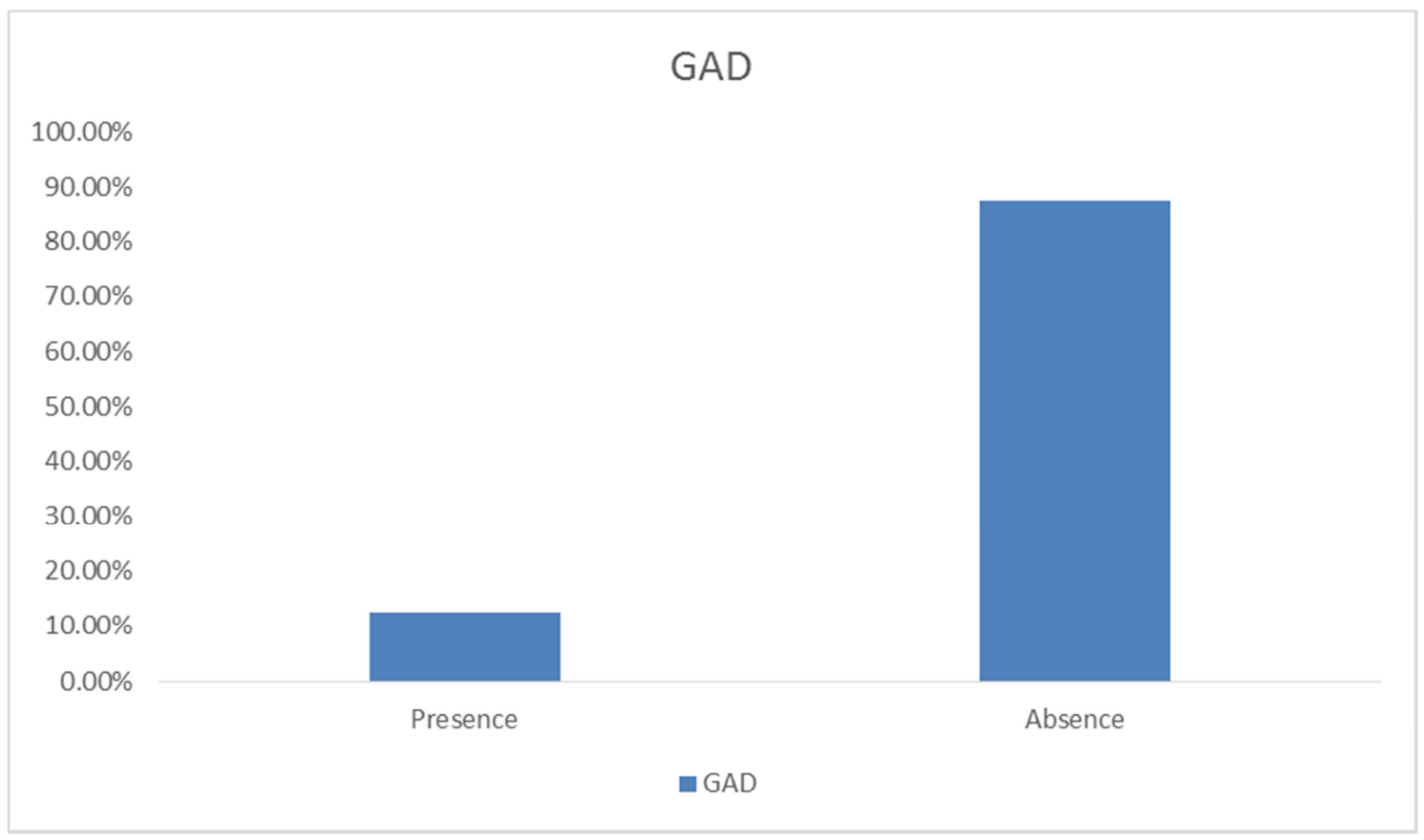

Figure 3. Respondents behaviour towards GAD.

\subsection{Association Between the Respondents' \\ Socio-demographic Characteristics and Their \\ Knowledge, Behavioural Response and ANXIETY}

Table 5, presents the association between the respondents' socio-demographic characteristics and their Knowledge, behavioural response and anxiety towards COVID-19 pandemic. As shown in table 5, there was positive significant association between Gender, age, level of education, chronic illness and occupation with knowledge of Respondents on
COVID-19 (p-value $<0.05$ ). Similarly, marital status, children in family, presence of chronic illness, occupation and taking avoidance behaviour was found to be significantly associated with GAD whereas only gender $(\mathrm{p}$-value $=0.001)$ and presence of chronic illness ( $p$-value $=0.008)$ was found to be associated with taking avoidance behaviour. There was no significant association of different variables with taking recommended behaviour was found.

Table 5. Association between the respondents'Socio-demographic Characteristics and their Knowledge, Behavioural response and Anxiety.

\begin{tabular}{|c|c|c|c|c|c|}
\hline \multirow{2}{*}{ Variables } & & \multicolumn{2}{|l|}{ Knowledge } & \multirow{2}{*}{ P-value } & \multirow{2}{*}{ GAD } \\
\hline & & Yes & No & & \\
\hline \multirow{4}{*}{ Gender } & Male & $166(85.1 \%)$ & $29(14.9 \%)$ & \multirow{4}{*}{$.025 *$} & $20(10.3 \%)$ \\
\hline & female & $143(76.1 \%)$ & $45(23.9 \%)$ & & $28(14.9 \%)$ \\
\hline & $18-24$ & $154(76.6 \%)$ & $47(23.4 \%)$ & & $25(12.4 \%)$ \\
\hline & $25-34$ & $138(87.3 \%)$ & $20(12.7 \%)$ & & $15(9.5 \%)$ \\
\hline \multirow[t]{3}{*}{ Age } & $35-44$ & $10(90.9 \%)$ & $1(9.1 \%)$ & \multirow[t]{3}{*}{$.005 *$} & $4(36.4 \%)$ \\
\hline & $45-54$ & $4(80.0 \%)$ & $1(20.0 \%)$ & & $2(40.0 \%)$ \\
\hline & $55-64$ & $3(37.5 \%)$ & $5(62.5 \%)$ & & $2(25.0 \%)$ \\
\hline \multirow{2}{*}{ Ethnicity } & Brahmin/ Chhetri & $254(82.5 \%)$ & $54(17.5 \%)$ & \multirow{2}{*}{.072} & $38(12.3 \%)$ \\
\hline & Other than Brahmin/ Chhetri & $55(73.3 \%)$ & $20(26.7 \%)$ & & $10(13.3 \%)$ \\
\hline \multirow{5}{*}{ Education } & Primary & $6(75.0 \%)$ & $2(25.0 \%)$ & \multirow{3}{*}{$.002 *$} & $3(37.5 \%)$ \\
\hline & Secondary & $44(62.9 \%)$ & $26(37.1 \%)$ & & $11(15.7 \%)$ \\
\hline & Graduate & $198(83.9 \%)$ & $38(16.1 \%)$ & & $23(9.7 \%)$ \\
\hline & Post-graduate & $57(89.1 \%)$ & $7(10.9 \%)$ & \multirow{4}{*}{.803} & $9(14.1 \%)$ \\
\hline & Doctorate & $4(80.0 \%)$ & $1(20.0 \%)$ & & $2(40.0 \%)$ \\
\hline \multirow{2}{*}{ Marital status } & Married & $71(81.6 \%)$ & $16(18.4 \%)$ & & $17(19.5 \%)$ \\
\hline & Unmarried & $238(80.4 \%)$ & $58(19.6 \%)$ & & $31(10.5 \%)$ \\
\hline \multirow{2}{*}{ Children } & Yes & $26(74.3 \%)$ & $9(25.7 \%)$ & \multirow{2}{*}{.315} & $12(34.3 \%)$ \\
\hline & No & $283(81.3 \%)$ & $65(18.7 \%)$ & & $36(10.3 \%)$ \\
\hline \multirow{2}{*}{ Chronic Illness } & Yes & $13(54.2 \%)$ & $11(45.8 \%)$ & \multirow{2}{*}{$.001 *$} & 9 \\
\hline & No & $296(82.5 \%)$ & $63(17.5 \%)$ & & $39(10.9 \%)$ \\
\hline \multirow{5}{*}{ Occupation } & Student & $183(78.9 \%)$ & $49(21.1 \%)$ & \multirow{5}{*}{$043 *$} & $27(11.6 \%)$ \\
\hline & Private job & $72(90.0 \%)$ & $8(10.0 \%)$ & & $4(5.0 \%)$ \\
\hline & Government job & $15(83.3 \%)$ & $3(16.7 \%)$ & & $5(27.8 \%)$ \\
\hline & Business & $14(87.5 \%)$ & $2(12.5 \%)$ & & $6(37.5 \%)$ \\
\hline & 0thers & $25(67.6 \%)$ & $12(32.4 \%)$ & & $6(16.2 \%)$ \\
\hline
\end{tabular}




\begin{tabular}{llllll}
\hline \multirow{2}{*}{ Variables } & & Knowledge & Po & P-value & GAD \\
\cline { 3 - 5 } & & Yes & - & $38(12.3 \%)$ \\
Knowledge on COVID-19 & Yes & - & - & $10(13.5 \%)$ \\
\multirow{2}{*}{ Avoidance behaviour } & No & Yes & - & - & $40(14.9 \%)$ \\
\multirow{2}{*}{ Recommended behaviour } & No & Yes & - & - & $8(7.0 \%)$ \\
& No & - & - & $48(12.7 \%)$ \\
\end{tabular}

Table 5. Continued.

\begin{tabular}{|c|c|c|c|c|c|c|}
\hline \multirow{2}{*}{ Variables } & & \multirow{2}{*}{ GAD } & \multirow{2}{*}{ P-value } & \multicolumn{2}{|l|}{$\mathbf{A B}$} & \multirow{2}{*}{ P-value } \\
\hline & & & & Yes & No & \\
\hline \multirow{2}{*}{ Gender } & Male & $175(89.7 \%)$ & \multirow{2}{*}{.171} & $119(61.0 \%)$ & $76(39.0 \%)$ & \multirow{2}{*}{$<0.001^{*}$} \\
\hline & female & $160(85.1 \%)$ & & $150(79.8 \%)$ & $38(20.2 \%)$ & \\
\hline \multirow{4}{*}{ Age } & $18-24$ & $176(87.6 \%)$ & \multirow{4}{*}{$0.066^{*}$} & $150(74.6 \%)$ & $51(25.4 \%)$ & \multirow{4}{*}{0.059} \\
\hline & $25-34$ & $143(90.5 \%)$ & & $100(63.3 \%)$ & $58(36.7 \%)$ & \\
\hline & $35-44$ & $7(63.6 \%)$ & & $8(72.7 \%)$ & $3(27.3 \%)$ & \\
\hline & $45-54$ & $3(60.0 \%)$ & & $5(100.0 \%)$ & $0(0.00 \%)$ & \\
\hline \multirow{2}{*}{ Ethnicity } & Brahmin/ Chhetri & $270(87.7 \%)$ & \multirow{2}{*}{.815} & $215(69.8 \%)$ & $93(30.2 \%)$ & \multirow{2}{*}{.709} \\
\hline & Other than Brahmin/ Chhetri & $65(86.7 \%)$ & & $54(72.0 \%)$ & $21(28.0 \%)$ & \\
\hline \multirow{5}{*}{ Education } & Primary & $5(62.5 \%)$ & \multirow{3}{*}{.087} & $6(75.0 \%)$ & $2(25.0 \%)$ & \multirow{3}{*}{.530} \\
\hline & Secondary & $59(84.3 \%)$ & & $44(62.9 \%)$ & $26(37.1 \%)$ & \\
\hline & Graduate & $213(90.3 \%)$ & & $172(72.9 \%)$ & $64(27.1 \%)$ & \\
\hline & Post-graduate & $55(85.9 \%)$ & \multirow{3}{*}{$025^{*}$} & $43(67.2 \%)$ & $21(32.8 \%)$ & \multirow{3}{*}{.103} \\
\hline & Doctorate & $3(60.0 \%)$ & & $4(80.0 \%)$ & $1(20.0 \%)$ & \\
\hline Marital status & Married & $70(80.5 \%)$ & & $55(63.2 \%)$ & $32(36.8 \%)$ & \\
\hline \multirow{2}{*}{ Children } & Yes & $23(65.7 \%)$ & \multirow{2}{*}{$<0.001$} & $25(71.4 \%)$ & $10(28.6 \%)$ & \multirow{2}{*}{.871} \\
\hline & No & $312(89.7 \%)$ & & $244(70.1 \%)$ & $104(29.9 \%)$ & \\
\hline \multirow{2}{*}{ Chronic Illness } & Yes & 15 & \multirow{2}{*}{$<0.001^{*}$} & 22 & 2 & \multirow{2}{*}{$.008^{*}$} \\
\hline & No & $320(89.1 \%)$ & & $247(68.8 \%)$ & $112(31.2 \%)$ & \\
\hline \multirow{5}{*}{ Occupation } & Student & $205(88.4 \%)$ & \multirow{5}{*}{$.004 *$} & $167(72.0 \%)$ & $65(28.0 \%)$ & \multirow{5}{*}{.525} \\
\hline & Private job & $76(95.0 \%)$ & & $58(72.5 \%)$ & $22(27.5 \%)$ & \\
\hline & Government job & $13(72.2 \%)$ & & $10(55.6 \%)$ & $8(44.4 \%)$ & \\
\hline & Business & $10(62.5 \%)$ & & $10(62.5 \%)$ & $6(37.5 \%)$ & \\
\hline & 0thers & $31(83.8 \%)$ & & $24(64.9 \%)$ & $13(35.1 \%)$ & \\
\hline Knowledge on & Yes & $271(87.7 \%)$ & 777 & $211(68.3 \%)$ & $98(31.7 \%)$ & 088 \\
\hline COVID-19 & No & $64(86.5 \%)$ & .771 & $58(78.4 \%)$ & $16(21.6 \%)$ & .088 \\
\hline Avoidance & Yes & $229(85.1 \%)$ & $034 *$ & - & - & - \\
\hline behaviour & No & $106(93.0 \%)$ & .034 & - & - & - \\
\hline Recommended & Yes & $331(87.3 \%)$ & 200 & - & - & - \\
\hline behaviour & No & $4(100.0 \%)$ & .299 & - & - & - \\
\hline
\end{tabular}

(* its shows that the variables are statistically significant).

\subsection{Multivariate Logistic Regression Analysis}

Table 6 shows the multivariate logistic regression analysis. Those respondents who had carried out avoidance behaviour were more likely to have GAD ( $\mathrm{AOR}=2.128,95 \% \mathrm{CI}$ : 1.0 4.957) similarly who had children were more likely to develop GAD (AOR=3.816, 95\% CI: 1.013- 14.378) and who had serious illness had a significantly higher level of anxiety than participants who had not $(\mathrm{AOR}=3.004,95 \% \mathrm{CI}$ : 1.0- 9.290). Female were more likely to take avoidance behaviour than men $(\mathrm{AOR}=2.704,95 \% \mathrm{CI}: 1.663-4.399)$ similarly adoption of the avoidance preventive behaviour was 5 times higher among the respondent with chronic illness as compare to those who had not $(\mathrm{AOR}=5.512,95 \% \mathrm{CI}$ : 1.116 27.210).

Table 6. Multiple Logistic Regression Analysis.

\begin{tabular}{|c|c|c|c|}
\hline Variable & Knowledge of COVID-19 AOR $(95 \% C I)$ & GAD AOR $(95 \% C I)$ & AB AOR $(95 \% C I)$ \\
\hline \multicolumn{4}{|l|}{ Gender } \\
\hline Male & 1.00 & 1.00 & 1.00 \\
\hline Female & $.574(.331-.996)$ & $1.509(.767-2.970)$ & $2.704(1.663-4.399)$ \\
\hline \multicolumn{4}{|l|}{ Age } \\
\hline$<35$ & $689(.133-3.579)$ & $1.513(.340-6.738)$ & $.569(.136-2.378)$ \\
\hline$\geq 35$ & 1.00 & 1.00 & 1.00 \\
\hline \multicolumn{4}{|l|}{ Ethnicity } \\
\hline Others & 1.00 & 1.00 & \\
\hline
\end{tabular}




\begin{tabular}{|c|c|c|c|}
\hline Variable & Knowledge of COVID-19 AOR $(95 \% C I)$ & GAD AOR $(95 \% C I)$ & AB AOR $(95 \% C I)$ \\
\hline \multicolumn{4}{|l|}{ Education } \\
\hline Primary/secondary & $.253(.101-.638)$ & $1.037(.399-2.694)$ & $.511(.238-1.095)$ \\
\hline Graduate & $.738(.312-1.744)$ & $.562(.245-1.290)$ & $.990(.530-1.847)$ \\
\hline Post graduate and more & 1.00 & 1.00 & 1.00 \\
\hline \multicolumn{4}{|l|}{ Marital status } \\
\hline Yes & $1.314(.515-3.351)$ & $1.012(.336-3.050)$ & $.413(.207-.824)$ \\
\hline No & 1.00 & 1.00 & 1.00 \\
\hline \multicolumn{4}{|l|}{ Children } \\
\hline Yes & $.875(.203-3.770)$ & $3.816(1.013-14.378)$ & $1.265(.401-3.993)$ \\
\hline No & 1.00 & 1.00 & 1.00 \\
\hline \multicolumn{4}{|l|}{ Chronic illness } \\
\hline Yes & $.212(.073-.611)$ & $3.004(1.0-9.290)$ & $5.512(1.116-27.210)$ \\
\hline No & 1.00 & 1.00 & 1.00 \\
\hline \multicolumn{4}{|l|}{ Occupation } \\
\hline Student & $.856(.438-1.671)$ & $1.111(.492-2.509)$ & $.976(.569-1.676)$ \\
\hline Other than student & 1.00 & 1.00 & 1.00 \\
\hline \multicolumn{4}{|l|}{ Knowledge } \\
\hline Yes & & $1.400(.601-3.264)$ & $.673(.350-1.294)$ \\
\hline No & & 1.00 & 1.00 \\
\hline \multicolumn{4}{|l|}{ Avoidance Behaviours } \\
\hline Yes & & $2.128(1.0-4.957)$ & \\
\hline No & & 1 & \\
\hline
\end{tabular}

(Notes: GAD, generalized anxiety disorder; $A O R$, adjusted odds ratio; 95\% $C I, 95 \%$ confidence interval; AB, Avoidance behaviour).

\section{Discussion}

Our web-based study shows relatively high percentage of respondents taking avoidance and recommended behaviour with a relatively low prevalence of GAD in the Nepalese public during COVID-19 outbreak. Anxiety symptoms were more likely to occur in respondent taking avoidance behaviour similarly those who had children as compare to those who were single without children and those with chronic illness. Female were more likely to take avoidance behaviour as compare to male and with the chronic illness were more likely to practice avoidance behaviour as compare to other.

Our data show that all of the respondents take at least one avoidance behaviour with $99 \%$ taking at least one of the recommended behaviour which is more than the study conducted on UK during COVID-19 outbreak [8]. In our study majority i.e. $80.7 \%$ of the respondent had better knowledge on COVID-19 with $87 \%$ of them correct rate on knowledge questionnaire which was similar with the study conducted in China during COVID-19 outbreak [9]. We found that $12.5 \%$ participants showed GAD which was lower than among the Chinese during COVID-19 pandemic [10] and was not different between male and female during COVID-19 outbreak which was similar with the study conducted in China during COVID-19 outbreak [10]. However, the result different from research in china that women were more likely to have anxiety than men $[11,12]$. GAD do not differ with age which differ to those of a previous study in China where Younger participants $(<35$ years) were more likely to develop anxiety than older participants ( $\geq 35$ years) during COVID-19 outbreak [10].

Respondents who had carried out avoidance behaviour had a significantly higher level of anxiety than participants who had not which is consistent with the study conducted in UK (England, Scotland, and Wales) during swine flu outbreak [10] and study conducted in USA during the swine flu epidemic in 2009 [14]. Women are to some extend more likely to adopt avoidance behaviour as compare to male which is consistent with the study conducted in UK on COVID-19 outbreak and study on swine-flu in USA $[8,14,15]$ however it differ with the study conducted in UK during swine flu epidemic where avoidance behaviour does not differ between male and female [13]. Respondent with the chronic illness were more likely to practice avoidance behaviour as compare to other which was inconsistent with the study conducted in UK during swine-flu outbreak [4].

\section{Conclusion}

It was found that COVID 19 pandemic has developed anxiety among adult population of Nepal. As knowledge score of COVID-19 was not associated with the preventive practices of the people which shows that the COVID 19 pandemic knowledge should be improved to reduce the anxiety level among the individual.

\section{Consent for Publication}

Not applicable.

\section{Availability of Data and Materials}

The dataset produced during this research is not available publicly due to privacy policy of the human subject involved in the study but can be made available from the corresponding author on reasonable request. 


\section{Acknowledgements}

We would like to acknowledge Nepal Health Research Council for providing ethical clearance for conducting this research work efficiently.

\section{References}

[1] Roy D, Tripathy S, Kar SK, Sharma N, Verma SK, Kaushal V. Study of knowledge, attitude, anxiety \& perceived mental healthcare need in Indian population during COVID-19 pandemic. Asian J Psychiatr. 2020.

[2] Liu X, Luo WT, Li Y, Li CN, Hong ZS, Chen HL, et al. Psychological status and behavior changes of the public during the COVID-19 epidemic in China. Infect Dis Poverty. 2020; 9 (1): 1-11.

[3] Ha T, Schensul S, Lewis J, Brown S. Early assessment of knowledge, attitudes, anxiety and behavioral adaptations of Connecticut residents to COVID-19. medRxiv. 2020.

[4] Rubin GJ, Amlôt R, Page L, Wessely S. Public perceptions, anxiety, and behaviour change in relation to the swine flu outbreak: Cross sectional telephone survey. BMJ. 2009; 339 (7713): 156.

[5] Tong, X., An, D., McGonigal, A., Park, S. P., Zhou D. Validation of the Generalized 44 All rights reserved. No reuse allowed without permission. (which was not certified by peer review) is the author/funder, who has granted medRxiv a license to display the preprint in perpetuity. The copyright holder for thi. Epilepsy Res. 2016; 120: 31-6.

[6] Spitzer RL, Kroenke K, Williams JBW, Löwe B. A brief measure for assessing generalized anxiety disorder: The GAD7. Arch Intern Med. 2006.
[7] Wang J, Mann F, Lloyd-Evans B, Ma R, Johnson S. Associations between loneliness and perceived social support and outcomes of mental health problems: A systematic review. BMC Psychiatry. 2018; 18 (1): 1-16.

[8] Atchison CJ, Bowman L, Vrinten C, Redd R, Pristera P, Eaton $\mathrm{JW}$, et al. Perceptions and behavioural responses of the general public during the COVID-19 pandemic: A crosssectional survey of UK Adults. medRxiv. 2020.

[9] Deye, N., Vincent, F., Michel, P., Ehrmann, S., Da Silva, D., Piagnerelli, M. Knowledge, attitude, and practices of health care professionals regarding pharmacovigilance in Pakistan. Value Heal. 2016; 152: 32-48.

[10] Huang Y, Zhao N. Generalized anxiety disorder, depressive symptoms and sleep quality during COVID-19 outbreak in China: a web-based cross-sectional survey. Psychiatry Res. 2020 .

[11] GAO, W., PING, S. \& LIU X. Gender differences in depression, anxiety, and stress among college students: a longitudinal study from China. J Affect Disord. 2020; 263: 292-300.

[12] Guo C., Yang J., Wei J, Li Y, Xu J, Jiang Y. Antioxidant activities of peel, pulp and seed fractions of common fruits as determined by FRAP assay. NutriRes. 1994; 23: 1719-1726.

[13] G. J. R, R. A, L. P, S. W. Public perceptions, anxiety, and behaviour change in relation to the swine flu outbreak: Cross sectional telephone survey. BMJ. 2009.

[14] Kanadiya MK, Sallar AM. Preventive behaviors, beliefs, and anxieties in relation to the swine flu outbreak among college students aged 18-24 years. J Public Health (Bangkok). 2011.

[15] Matud MP. Gender differences in stress and coping styles. Personality and individual differences. 2004 Nov 1; 37 (7): 1401-15. 